\title{
Novel mutations in TPM2 and PIEZO2 are responsible for distal arthrogryposis (DA) 2B and mild DA in two Chinese families
}

\author{
Shan $\mathrm{Li}^{1+}$, Yi You ${ }^{1 \dagger}$, Jinsong Gao ${ }^{2}$, Bin Mao ${ }^{1}$, Yixuan Cao ${ }^{1}$, Xiuli Zhao ${ }^{1 *}$ and Xue Zhang ${ }^{1 *}$
}

\begin{abstract}
Background: Distal arthrogryposis (DA) is a group of clinically and genetically heterogeneous disorders that involve multiple congenital limb contractures and comprise at least 10 clinical subtypes. Here, we describe our findings in two Chinese families: Family 1 with DA2B (MIM 601680) and Family 2 with mild DA.

Methods: To map the disease locus, two-point linkage analysis was performed with microsatellite markers closed to TPM2, TNNI2/TNNT3 and TNNC2. In Family 1, a positive LOD (logarithm of odds) score was only obtained at the microsatellite marker close to TPM2 and mutation screening was performed using direct sequencing of TPM2 in the proband. In Family 2, for the LOD score that did not favor linkage to any markers, whole-exome sequencing (WES) was performed on the proband. PCR-restriction fragment length polymorphism (RFLP) and bioinformatics analysis were then applied to identify the pathogenic mutations in two families. In order to correlate genotype with phenotype in DA, retrospective analyses of phenotypic features according to the TPM2 and PIEZO2 mutation spectrums were carried out.

Results: A heterozygous missense mutation c.308A > G (p.Q103R) in TPM2 in Family 1, and a novel variation c.8153G > A (p.R2718Q) in PIEZO2 in Family 2 were identified. Each of the two novel variants was co-segregated with the DA manifestations in the corresponding family. Bioinformatics analysis from several tools supported the pathogenicity of the mutations. Furthermore, our study suggests that there is no relation between the types or locations of TPM2 mutations and the clinical characteristics, and that different inheritance modes and mutation types concerning PIEZO2 cause distinct clinical manifestations.
\end{abstract}

Conclusions: We report two novel mutations within TPM2 and PIEZO2 responsible for DA2B and mild DA in two Chinese families, respectively. Our study expands the spectrum of causal mutations in the TPM2 and PIEZO2 genes.

Keywords: Distal arthrogryposis, TPM2, PIEZO2, Novel mutation, Genotype-phenotype

\section{Background}

Distal arthrogryposis (DA) is a group of rare autosomal-dominant limb disorders that involve congenital contractures of two or more body areas without primary neurologic or muscular disease [1]. DA is clinically and genetically heterogeneous and comprises more than 10 clinical subtypes $(1,2 \mathrm{~A}, 2 \mathrm{~B}, 3-10)$ based on additional phenotypic features [2]. The most common

\footnotetext{
* Correspondence: xiulizhao@ibms.pumc.edu.cn; xuezhang@pumc.edu.cn tShan Li and Yi You contributed equally to this work.

${ }^{1}$ Department of Medical Genetics, Institute of Basic Medical Sciences, Chinese Academy of Medical Sciences \& School of Basic Medicine, Peking Union Medical College, 5 Dong Dan San Tiao, Dong Cheng District, Beijing 100005, China

Full list of author information is available at the end of the article
}

subtypes are DA1 and DA2B [3]. The hallmarks of DA1 include camptodactyly and clubfoot, without additional abnormalities [4]. Patients with DA2B typically present severe camptodactyly, triangular face, prominent nasolabial folds, attached earlobes, downward-slanting palpebral fissures, small mouth, and prominent chin [5]. DA3 (Gordon syndrome, MIM 114300) is distinguished from other DA by short stature and cleft palate [6]. A small subset of DA5 (MIM 615065) is known by the presence of ocular abnormalities, typically ptosis, ophthalmoplegia, and/or strabismus, in addition to contractures of the skeletal muscles [2]. To date, mutations in TPM2, TNNI2, TNNT3, and MYH3 which encode components of the contractile apparatus of fast-twitch myofibers or

(c) The Author(s). 2018 Open Access This article is distributed under the terms of the Creative Commons Attribution 4.0 International License (http://creativecommons.org/licenses/by/4.0/), which permits unrestricted use, distribution, and 
embryonic myosin, have been implicated in DA1 and DA2B [5, 7]. The PIEZO2 gene encodes a mechanically activated cation channel, and the mutations of PIEZO2 have been reported in DA3, DA5 and Marden-Walker syndrome MWKS [8].

In the current study, we recruited two families affected by DA2B and mild DA, and conducted two-point linkage analysis and Sanger sequencing or/ and whole-exome sequencing (WES) to identify pathogenic mutations. Our findings indicated that a novel mutation in TPM2 was responsible for DA2B in Family 1 , and that a missense mutation in PIEZO2 was the etiology of mild arthrogryposis in Family 2 . This is the first finding that TPM2 and PIEZO2 are associated with DA in Chinese population, which provided new clues for the correlation between genotype and phenotype in DA.

\section{Methods}

Participants collection and isolation of genomic DNA

Two Chinese pedigrees with autosomal dominant hereditary DA were collected (Fig. 1). Careful examinations and clinical record reviews were performed for each patient to confirm the original diagnosis. Peripheral blood or cord blood samples were obtained from all participants. Genomic DNA was isolated from blood samples by the standard method of proteinase $\mathrm{K}$ and phenol/chloroform extraction [9]. All participants provided informed consent, and this study was approved by the ethics committee of the Peking Union Medical College Institutional Review Board.

\section{Linkage analysis}

Using the UCSC Genome Browser (http://genome.ucsc.edu), three microsatellite repeat sequences near TPM2 (chr9: 35,681,993-35,690,056), TNNI2 (chr11: 1,840,202-1,841,680)/ TNNT3 (chr11: 1,919,569-1,938,706), and TNNC2 (chr20: $45,823,216-45,827,314$ ) were selected as markers for two-point linkage analysis (Additional file 1: Table S1). PCR products were separated by conventional $8 \%$ polyacrylamide gel electrophoresis. Two-point linkage analysis was carried out using the MLINK program in the LINKAGE software package (version 5.2) [10]. The assumptions of the analysis were autosomal dominant inheritance, penetrance of $100 \%$, a disease allele frequency of 0.0001 , and equal recombination frequencies for males and females.

\section{Whole-exome sequencing}

In Family 2, exome capture was performed using an Agilent SureSelect Human All Exon kit (Agilent Technologies, Wilmington, DE), according to the manufacturer's instructions. DNA was broken into fragments of 180 280 bp using an ultrasonoscope. A sequencing-capture DNA library was prepared by means of a HiSeq 2000 platform (Illumina, San Diego, CA). Collection of primary data, including error assessment and base calling, was accomplished with an Illumina Pipeline (version 1.3.4) [11]. Sequencing reads were aligned to the human reference genome sequence from the UCSC database. Variants were filtered by the Single Nucleotide Polymorphism Database (dbSNPs; https://www.ncbi.nlm.nih.gov/projects/SNP/) and the 1000 Genomes database (www.internationalgenome.org). Reported disease-causing mutations were confirmed based on the Human Gene Mutation Database at the Institute of Medical Genetics in Cardiff (HGMD, http://www.hgmd.cf.ac.uk/ac/index.php) or previous literature. Functional domain was predicted by the SMART database (http://smart.embl-heidelberg.de).

\section{Validation of mutations}

For Family 1, TPM2 (the primary candidate gene) was amplified by PCR using 5 primer pairs (E1-2, E3-6, E79, E10, and E11; Additional file 1: Table S1). To validate the mutation identified in proband, co-segregation analysis in the family was applied by PCR-RFLP. PCR fragments amplified by primer E3F (forward) and In-E4R (reverse), which were digested by the restriction

Family 1
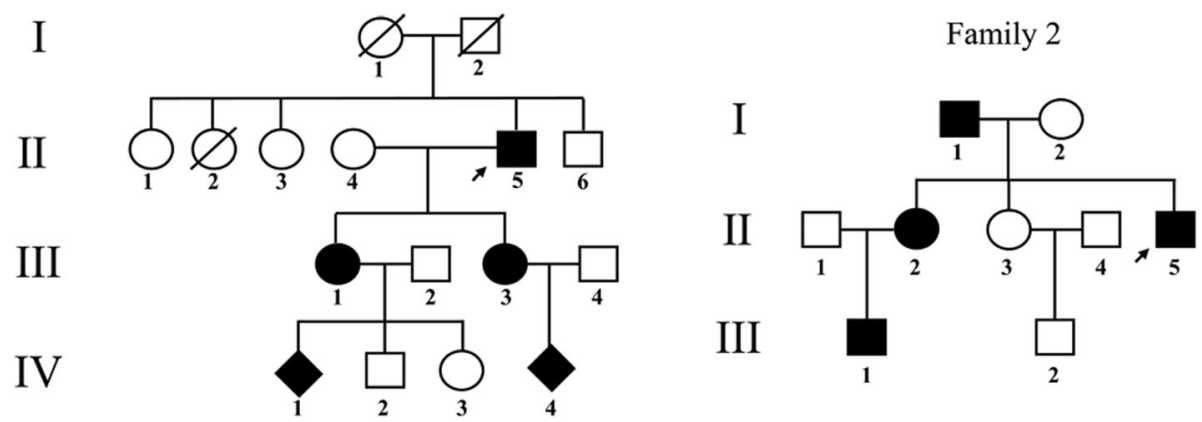

Fig. 1 Pedigrees of the two families with DA2B (Family 1) and mild DA1 (Family 2). Arrows indicate the probands in each family 
endonuclease Sma I, were separated by $2 \%$ agarose gel electrophoresis.

For Family 2, PCR and Sanger DNA sequencing were performed to verify the variant in PIEZO2 (Additional file 1 : Table S1). Amplicons from all available family members were analyzed by RFLP using Taq I and 8\% polyacrylamide gel electrophoresis.

\section{Bioinformatics analysis}

The amino acid sequences of the tropomyosin beta chain (TPM2; NP_001288156.1), component 2 of the piezo-type mechanosensitive ion channel (PIEZO2; NP_071351.2) and their homologues of 10 animal species were obtained from the NCBI (National Center for Biotechnology Information) protein database in FASTA format. Multiple-sequence alignment and conservation analysis were performed with MEGA software (version 7; Institute for Genomics and Evolutionary Medicine, Temple University, Philadelphia, PA). The pathogenicity of missense variants was predicted by PolyPhen-2 (http://genetics.bwh.harvard.edu/pph2), Scale-Invariant Feature Transform (SIFT; http://sift.jcvi.org), Protein Variation
Effect Analyzer (PROVEAN; http://provean.jcvi.org/seq_submit.php), Mutation Taster (http://www.mutationtaster.org) and M-CAP (http://bejerano.stanford.edu/mcap). The mutant proteins translated from the missense variants in TPM2 and PIEZO2 were assessed using the crystal structure of contractile protein at $35 \AA$ resolution (PDB code $2 \mathrm{~W} 4 \mathrm{U}$ ) and metal transport protein at $4.8 \AA$ resolution (PDB code 3JAC), respectively. SWISS-MODEL was used to model the amino acid changes in 3D structure (SWISS-MODEL; http://swissmodel.expasy.org/). Hydrogen (H)-bonding interactions with neighboring residues were visualized with the Swiss-Pdb Viewer (version 4.10; Swiss Institute for Bioinformatics, Lausanne, Switzerland).

\section{Results}

Clinical evaluation

Family 1 was a four-generation pedigree involving DA of variable severity (Fig. 1). Four patients and a fetus of 22-week gestational age were recruited for phenotypic evaluation by clinical examination or by administration of
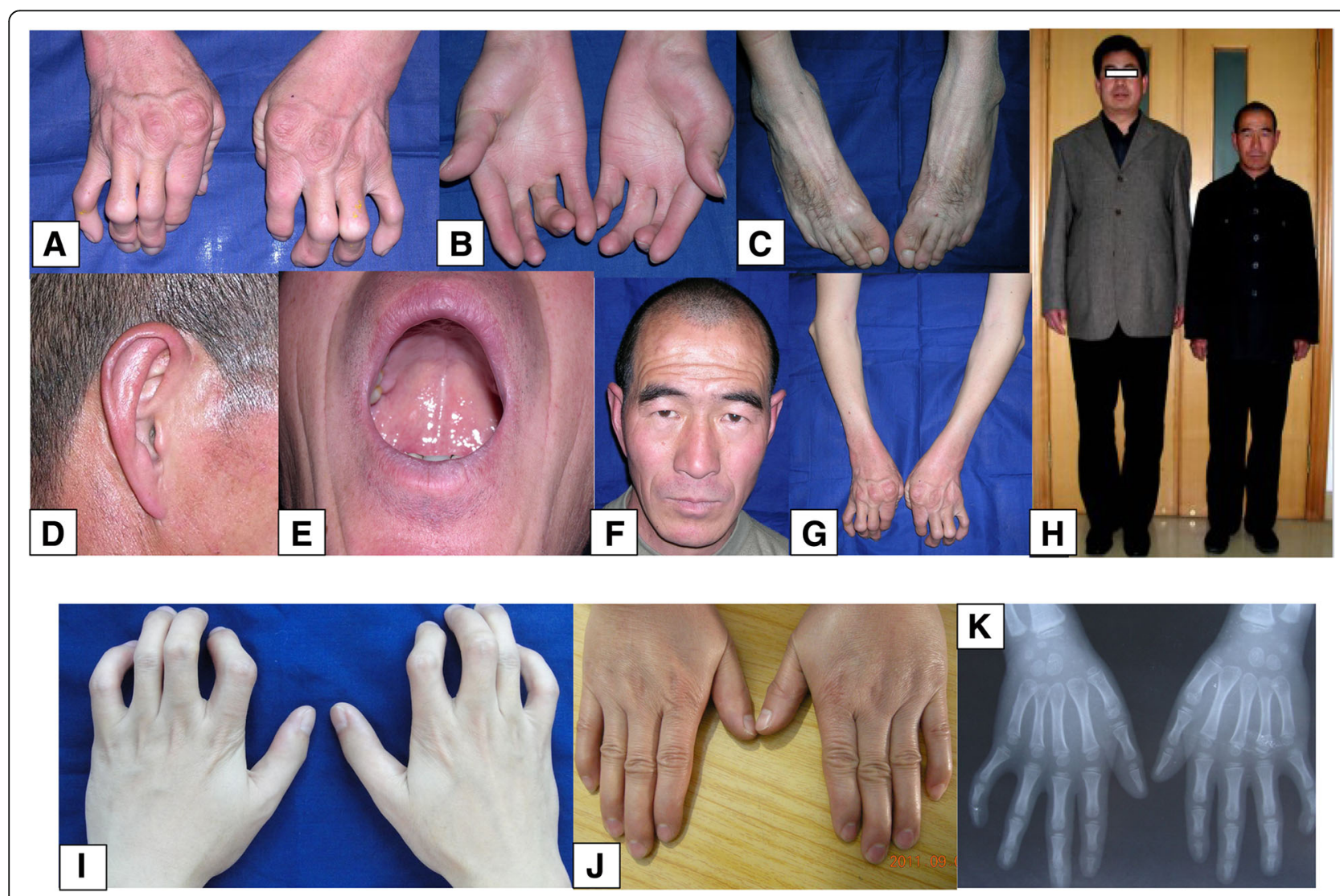

Fig. 2 Clinical features of patients in Family 1 with DA2B (a-h) and Family 2 with mild DA (i-k). a Ulnar deviation and contractures in patient II5. b Camptodactyly and adducted thumbs in patient III1. c Flexed toes and talipes equinovarus in patient II5. $\mathbf{d}$ Dysplastic ear and attached ear lobes in patient II5. e Small mouth with limited opening in patient II5. f Downward-slanting palpebral fissures, a small chin, and deep folds in the nasolabial area and forehead in patient II5. g Flexion contracture yielding stiff elbows in patient II5. $\mathbf{h}$ Short stature of patient II5 (unaffected II6 [left], $178 \mathrm{~cm}$; patient II5 [right], $156.5 \mathrm{~cm}$ ). i Camptodactyly and ulnar deviation in patient II5. $\mathbf{j}$ Mild contractures of the fingers in patient II2. $\mathbf{k}$ X-ray findings of patient III1 in 5 years old indicate camptodactyly and ulnar deviation 
a questionnaire. The proband (II5) was a 59-year-old male with bilateral and symmetric congenital contractures of the distal limbs, including severe ulnar deviation, camptodactyly, adducted thumbs and overlapping fingers (Fig. 2a, g). Short stature and minor facial anomalies, including a triangular face, downward-slanting palpebral fissures, and a small mouth, were also noted in the proband, but not in his two affected daughters (III1 and III3) (Fig. 2d-h). The proband had more severe phenotype than his two affected daughters did (Fig. 2b-c), indicating phenotypic variability in the family. Patient III1 was pregnant, whose fetus was diagnosed to have phenotype of DA on ultrasound examination. In particular, the ultrasound results indicated extended wrists, clenched fists, and bilateral club feet (Additional file 2: Figure S1). According to the clinical characteristic of the patients mentioned above, we concluded that this family was affected by D2AB (Table 1).

Family 2 was a three-generation pedigree with mild DA, and including four affected individuals and five unaffected family members (Fig. 1). The proband (II5) was a 37-year-old man, with congenitally bilateral-symmetric contractures in fingers 2-5 (Fig. 2i). The other three affected family members had mild contractures restricted to the distal phalanges (Fig. 2j-k). All the affected individuals presented variable arthrogryposis without other manifestations of DA3, DA2B, or DA5 such as cleft palate or ocular/facial abnormalities (Table 1).

\section{Linkage analysis}

For Family 1, two-point linkage analysis yielded a positive LOD score for 9p13AAC near TPM2, indicating linkage of the loci (Additional file 3: Table S2 and Fig. 3a). LOD scores for the other two markers near TNNI2/TNNT3 and TNNC2 did not favor linkage. Therefore, TMP2 was regarded as the candidate gene for Family 1. For Family 2, LOD scores indicated no linkage for any of the three locations.

Identification of a missense mutation in TPM2 in family 1 Sanger sequencing of the proband indicated the presence of a heterozygous missense mutation c.308A > G in

Table 1 Clinical phenotypes of patients with TPM2 and PIEZO2 gene mutations in two families

\begin{tabular}{|c|c|c|c|c|c|c|c|}
\hline & Family & & & Family & & & \\
\hline & $11-5$ & || $\mid-1$ & $111-3$ & $11-5$ & $\mid-1$ & $\|-2$ & || $\mid-1$ \\
\hline Age/Sex & $59 / M$ & $35 / F$ & $32 / F$ & $37 / M$ & $65 / M$ & $42 / F$ & $11 / \mathrm{M}$ \\
\hline Decreased facial expression & - & - & - & - & - & - & - \\
\hline Ptosis/Limited ocular motility & + & - & - & - & - & - & - \\
\hline Ear deformity & - & - & - & - & - & - & - \\
\hline Small pursed mouth & + & - & - & - & - & - & - \\
\hline Trismus & - & - & - & - & - & - & - \\
\hline Cleft palate & - & - & - & - & - & - & - \\
\hline Nasolabial folds & + & - & - & - & - & - & - \\
\hline Triangularly shaped face & + & - & - & - & - & - & - \\
\hline Finger contractures & + & + & + & + & + & + & + \\
\hline Camptodactyly & + & + & + & + & + & + & + \\
\hline Elbow contractures & + & - & - & - & - & - & - \\
\hline Limited wrist extension & + & - & - & - & - & - & - \\
\hline Asymmetric legs/feet & - & - & - & - & - & - & - \\
\hline Clubfeet & + & + & + & + & + & + & + \\
\hline Scoliosis & - & - & - & - & - & - & - \\
\hline Short stature & + & - & - & + & + & + & ND \\
\hline Muscle weakness & - & - & ND & - & - & - & - \\
\hline Sensorineural hearing loss & - & - & - & - & - & - & - \\
\hline Pain problems & - & - & - & - & - & - & - \\
\hline Surgical operations & + & + & ND & - & - & - & - \\
\hline Additional symptoms & - & - & ND & - & - & - & - \\
\hline Radiographs & - & - & - & + & - & - & + \\
\hline DA classification & $\mathrm{DA} 2 \mathrm{~B}$ & & & DA1 & & & \\
\hline Disease-causing mutation & TPM2: & & & PIEZOZ & & & \\
\hline
\end{tabular}

+,present;-, absent; NA information not available, ND not determined 


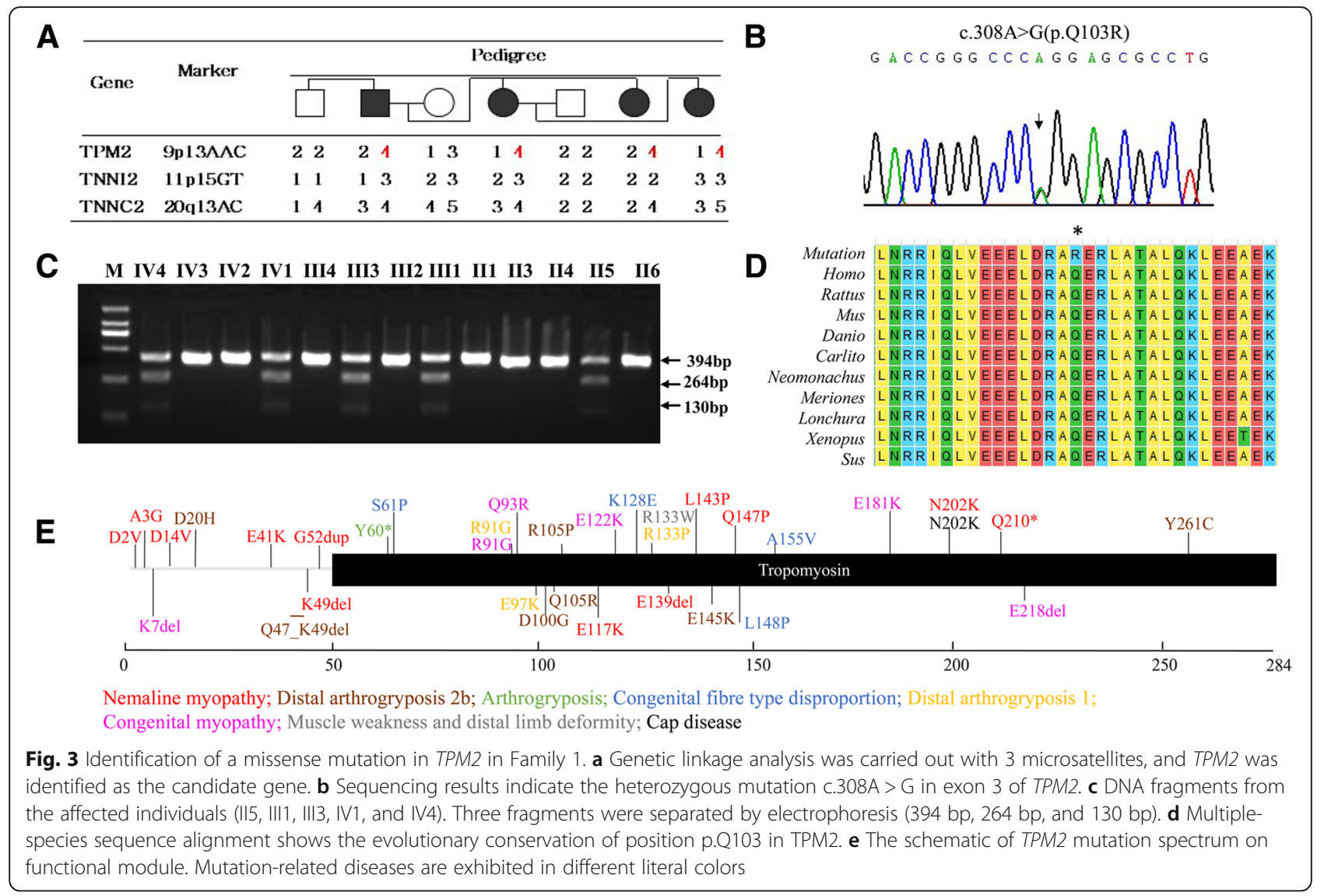

exon 3 (Fig. 3b). This mutation led to the substitution of glutamine for arginine at position 103 of the amino acid sequence (Q103R) and yielded a novel Sma I restriction site in the mutant TPM2 gene. PCR products digested by Sma I were separated by $2 \%$ agarose gel electrophoresis. All unaffected individuals had the expected $394 \mathrm{bp}$ fragments, whereas all affected members had 394 bp, $264 \mathrm{bp}$ and $130 \mathrm{bp}$ fragments (Fig. 3c).

Identification of a missense mutation in PIEZO2 in family 2 On average, 90\% coverage was achieved for exome regions from genomic DNA samples (II5) of Family 2, and the sequencing depth exceeded $100 \times$. A novel heterozygous variant was found in exon 52 of PIEZO2 in the proband. This mutation resulted in the substitution of glutamine to arginine (p.R2718Q) and abrogated the restriction site recognized by Taq I (Fig. 4a). Co-segregation in members of Family 2 was observed in PCR-RFLP results. The mutant-allele amplicon could not be digested by Taq I and yielded a $104 \mathrm{bp}$ fragment, whereas the wild-type-allele amplicon was digested by Taq I to produce $70 \mathrm{bp}$ and 34 bp fragments. All samples from affected members of Family 2 (II2, III1, II5, and I1) yielded three fragments (104 bp, $70 \mathrm{bp}$, and $34 \mathrm{bp}$ ); in contrast, all samples from unaffected members produced only two fragments (70 bp and $34 \mathrm{bp}$ ) (Fig. 4b).

\section{Results of bioinformatics analysis}

Conservation analysis of p.Q103 in TPM2 and p.R2718 in PIEZO2 among 10 species indicated that the two amino acid sites are highly conserved (Figs. 3d and 4c). There is no record about the frequency of the two mutations in ExAC browser, esp6500si, 1000 Genome, dbSNP, gnomAD browser, etc. Mutations p.Q103R in TPM2 and p.R2718Q in PIEZO2 were predicted to be potentially damaging by bioinformatics analysis from softwares SIFT, Polyphen2, MutationTaster, M-CAP, and PROVEAN programs. These prediction programs yielded similar outcomes regarding pathogenicity except that Polyphen 2 predicted p.Q103R a benign mutation. Therefore, substitution of wild-type residues might change the biological function. (Table 2).

According to the SWISS-MODEL prediction, substitution of a neutral amino acid (glutamine) to a basic amino acid (arginine) at position 103 of tropomyosin would likely influence the conformation and charge properties of the protein. In addition, the presence of arginine may alter the acidic surface properties of the protein and could potentially impair its molecular function (Fig. 5). Three 


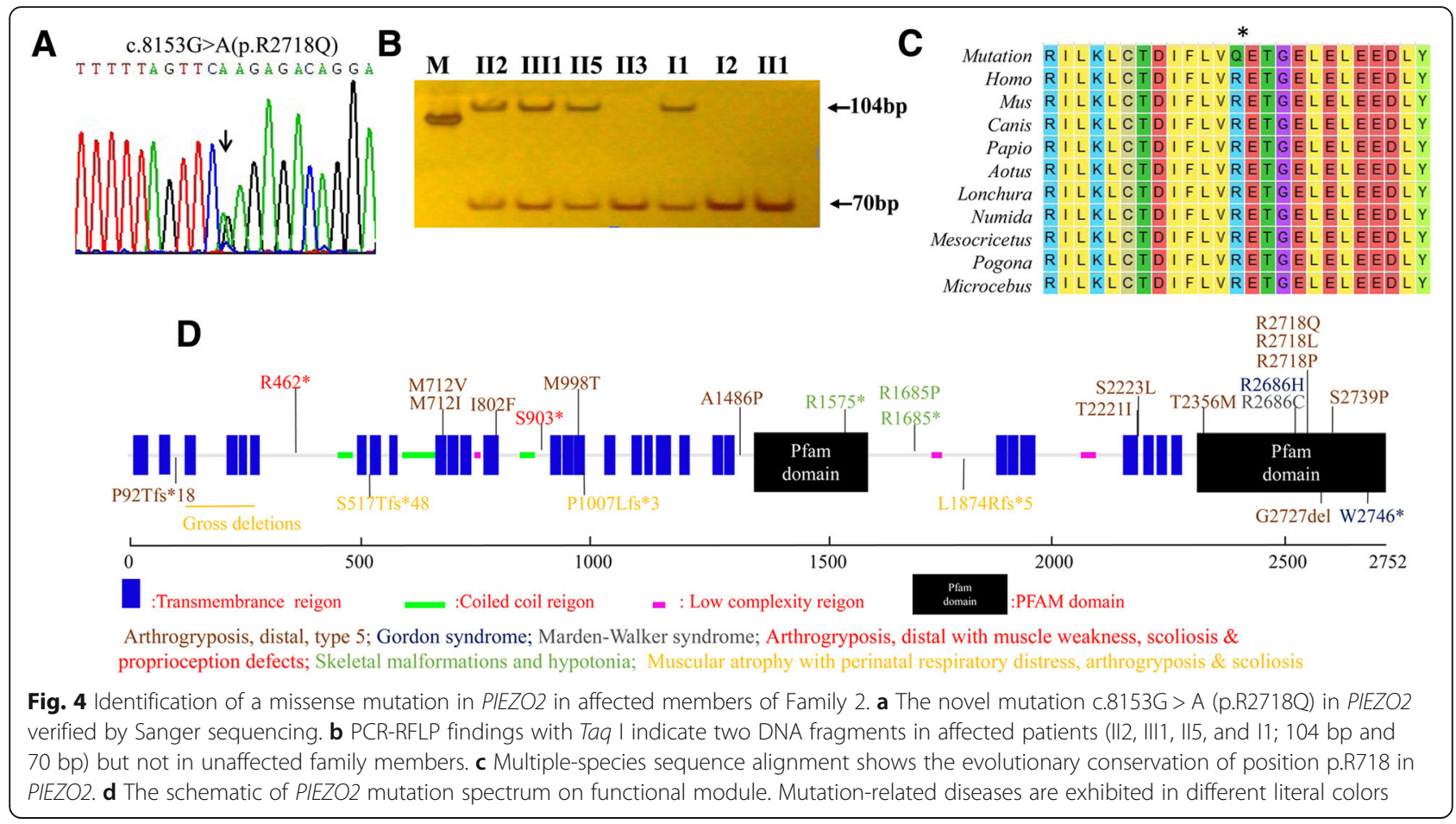

substitutions in position 2718 of component 2 of the piezo-type mechanosensitive ion channel were simulated by SWISS-MODEL and represented with Ribbon. This simulation predicted that R2718 interacted via H-bonding with residues D2713, T2712, F2715, G2721 and E2722. Substitution of p.R2718Q destroyed the H-bonding between R2718 and D2713 (Fig. 6b). This change could interfere with the protein function by conformational alteration.

\section{Genotype-phenotype correlations}

According to the mutation of TPM2 in this study and retrospective analysis of other related studies, we found there is no any significant clear correlation between types or location of TPM2 mutations and clinical manifestations (Fig. 3e).

The patients of Family 2 carrying the mutation p.R2718Q in PIEZO2 have mild arthrogryposis without other manifestations. It was interesting to note that the mutation p.R2718Q was located at the same site as two previously reported mutations (p.R2718P and p.R2718L). Although all the three mutations located at the same site, mutation p.R2718P and p.R2718L led to more severe phenotypes. We constructed three-dimensional models of the mutated proteins to investigate the possible molecular pathogenicity. We found that substitution of leucine tended to lose H-bond with D2713, and to disrupt the structure of neighbor $\alpha$-helical (Fig. 6c). On the contrary, substitution of proline did not affect the $\alpha$-helix, but destroyed the connection between H-bonds to T2712, D2713 and F2715 (Fig. 6d). We noticed that substitution of glutamine was prone to maintain the original H-bonds and keep the original molecular conformation (Fig. 6b), which enlighten the association between genotypes and phenotypes.

Further, analysis indicated that DA3, DA5, MWKS patients with dominant inheritance carried heterozygous mutations which are mainly localized at the C-terminal of PIEZO2, and that recessive inherited PIEZO2-related disorders are all caused by biallelic truncating mutations in PIEZO2 (Table 3) (Fig. 4d).

\section{Discussion}

DA is defined broadly as a heterogeneous category of inherited limb malformation syndromes with substantial

Table 2 Predictions of functional effects for two mutations in this study

\begin{tabular}{llllll}
\hline & SIFT & Polyphen-2 & MutationTaster & M-CAP & PROVEAN \\
\hline TPM2:c.308A > G(p.Q103R) & Damaging & Benign & Disease causing & Possibly Pathogenic & Deleterious \\
score & 0 & 0.06 & - & 0.349 & -3.13 \\
PIEZO2:c.8153G > A (p.R2718Q) & Damaging & Probably Damaging & Disease causing & Possibly Pathogenic & Deleterious \\
score & 0 & 1 & - & 0.821 & -3.35 \\
\hline
\end{tabular}



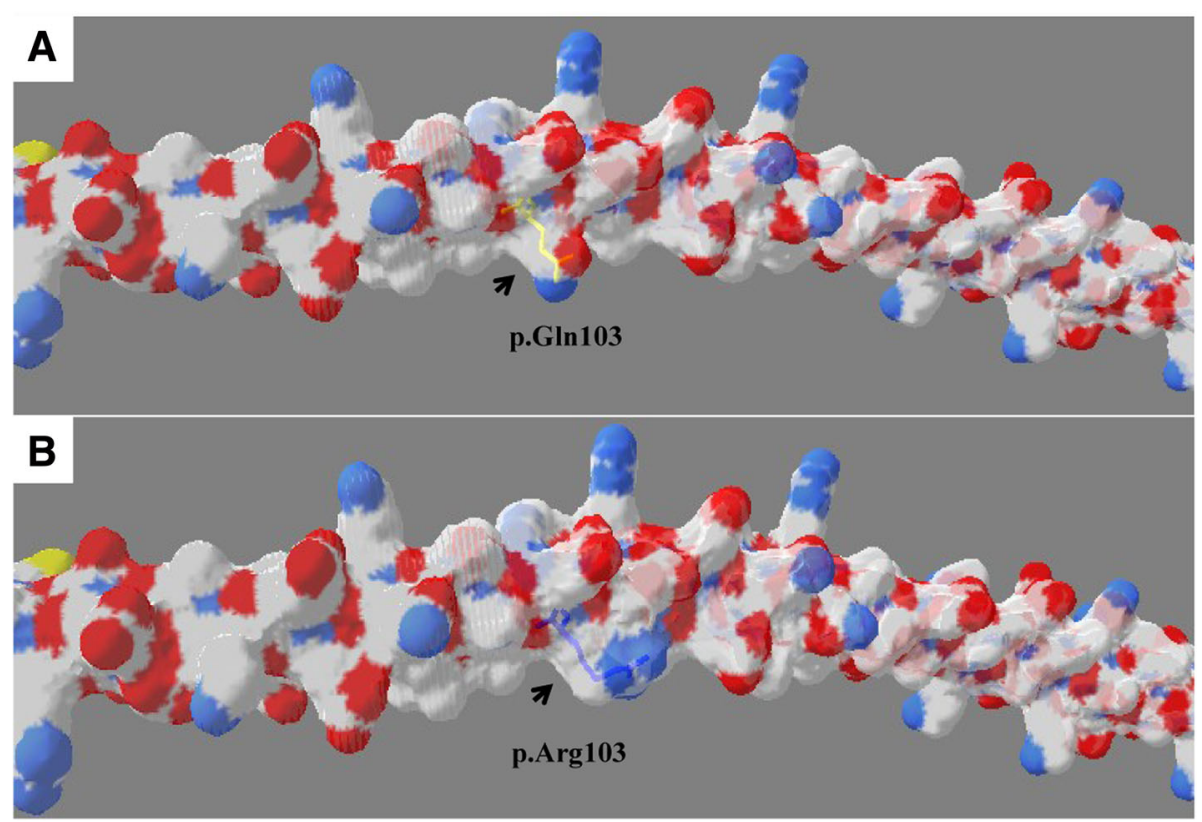

Fig. 5 SWISS-MODEL prediction of the p.Q103R mutation in tropomyosin. a The 3-dimensional structure of wild-type tropomyosin with position 103 indicated by a black arrow. Nearby acidic (red) and basic (blue) surface properties are illustrated with the neutral glutamine residue shown as a yellow line. $\mathbf{b}$ Acidic surface properties are destroyed with the substitution of basic arginine for glutamine. The substitution is depicted as a blue line

clinical and genetic heterogeneity and variable expressivity $[3,12,13]$. Here, we report two novel mutations within TPM2 and PIEZO2 responsible for DA2B and mild DA in two Chinese families, respectively.

Mutations in TPM2 were previously thought to solely cause DA1 [13]. However, mutations in TPM2 have recently been reported to be associated with DA2B $[14,15]$. In line herewith, our patients carrying a missense mutation p.Q103R in TPM2 presented typical DA2B as well. Our study supports that DA1 and DA2B may be categorized as phenotypic extremes of the same disorder rather than as different DA types [15]. Currently, HGMD (Professional 2018.1) shows that 35 disease-causing mutations within TPM2 cause 11 different myopathic conditions including nemaline myopathy, congenital myopathy, DA2B, DA1 and Cap disease [16-19]. The identified
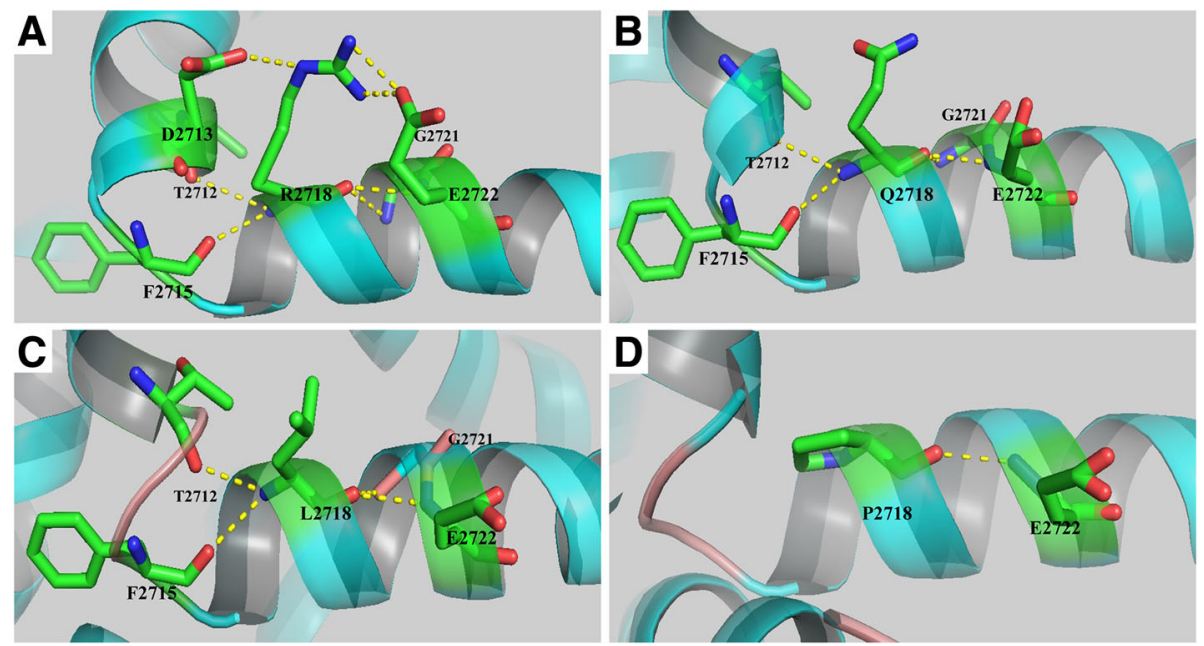

Fig. 6 Three substitutions in position 2718 of PIEZO2 are simulated by means of SWISS-MODEL and are represented with Ribbon. a The wild-type amino acid at position 2718 is depicted, with $\mathrm{H}$-bonds shown by yellow dashed lines. $\mathbf{b}$ The p.R2718Q substitution maintains the most parts of $\mathrm{H}$ bonding interactions. $\mathbf{c}$ Leucine tends to lose H-bond with D2713, and the nearby a-helical structure is disrupted. $\mathbf{d}$ Substitution with proline eliminates most of the $\mathrm{H}$-bonds with neighboring amino acids 


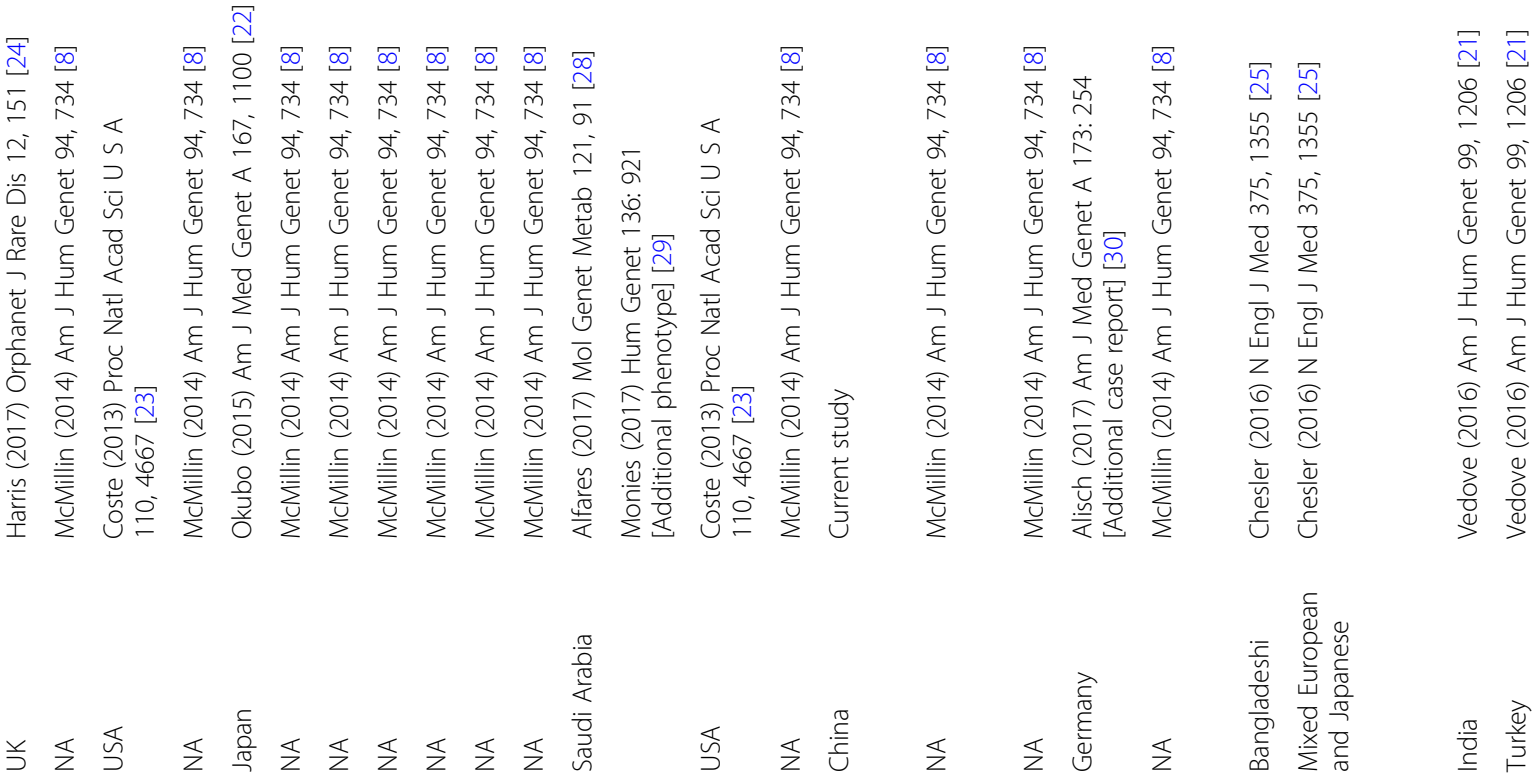

是是是是是是是是是是是是

\& $\&$ \&

电早东

妥采

产兴

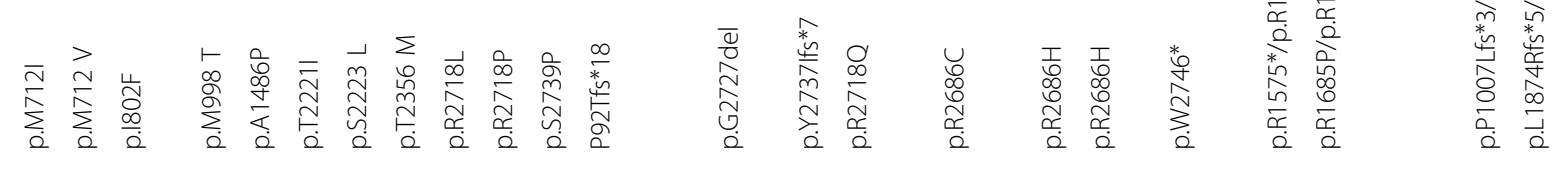

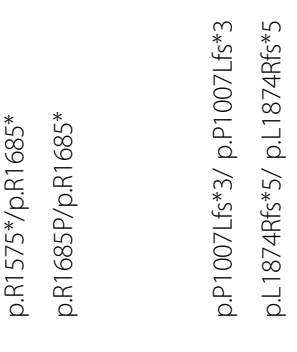




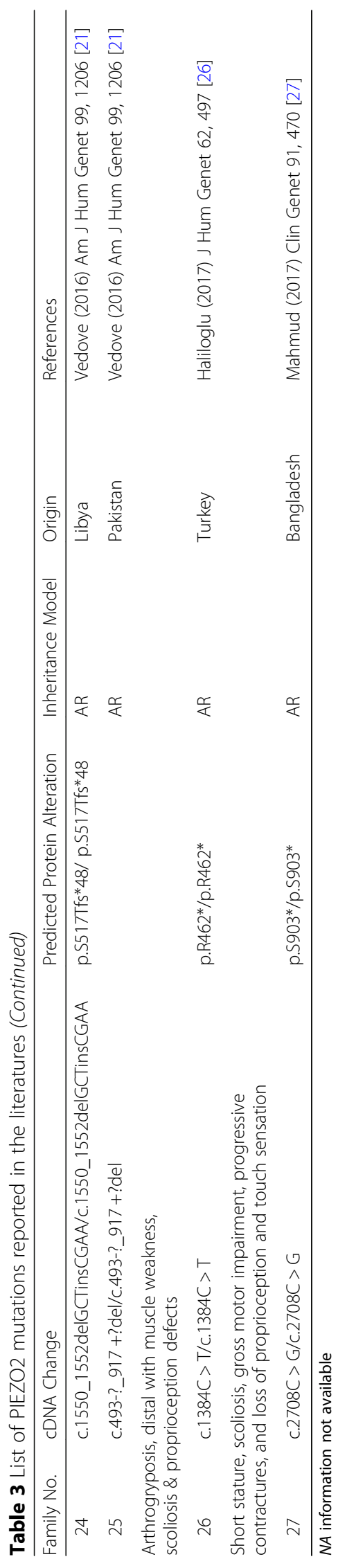


mutations were distributed across the TPM2 gene and cannot be mapped to any hotspots. The number of mutations related to DA1 and DA2B account for $25.7 \%$ (9/35) of all reported, most of which are missense mutations (8/9) (Fig. 3e). According to the mutation of TPM2 in this study and retrospective analysis of related studies, we found that there is not any obvious correlation between types or location of TPM2 mutations and clinical manifestations (Fig. 3e), as reported by Beck et al. and Marttila et al. [15, 20].

Mutations in PIEZO2 have been reported in Marden-Walker syndrome, DA3, DA5, and other DAs that involve in sense of touch damage and impaired proprioception. Piezo2 is a transmembrane protein, which can be found in many tissues, with a function of adapting mechanically activated currents. It composes of stretch-activated ion channels, and was reported to be associated with touch sensation [21]. Our study presents Chinese patients carrying a missense mutation p.R2718Q in PIEZO2 for the first time who have mild arthrogryposis without other manifestations. The mutation c.8153G > T (p.R2718L) in PIEZO2 was previously described in a family with a subtype of DA5 characterized by contractures, limited eye movements, restrictive lung disease, and variably absent cruciate knee ligaments [8]. McMillin et al. reported a male patient with DA5 in whom a heterozygous mutation c.8153G > $\mathrm{C}$ was identified in PIEZO2. This mutation yielded an arginine-to-proline (R2718P) substitution and induced the features of ptosis, ophthalmoplegia, scoliosis, and pulmonary disease [8]. Although the mutation site is the same for these two cases and the patients in this study, the substituted amino acid differs, and resulted various phenotypes, which may enlighten the association between genotypes and phenotypes. HGMD (Professional 2018.1) records 26 disease-causing mutations in PIEZO2 and 10 resulted disorders, and the DA5 is the most common condition (14/26) in PIEZO2-related disorders (Table 3) (Fig. 4d). Heterozygous gain-of-function (GoF) mutations in PIEZO2 have been reported in MWKS, DA3, DA5, which typically involve congenital contractures of hands and feet, or cleft palate, ophthalmoplegia, ptosis, and cerebellar malformations [8, 22-24]. Recent studies have discovered that biallelic loss-of-function (LoF) mutations in PIEZO2 cause muscular atrophy with perinatal respiratory distress, arthrogryposis, scoliosis and proprioception defects [21, 25-27]. Phenotype-genotype correlations analysis suggests that heterozygous mutations carried by the DA3, DA5, MWKS patients are mainly localized at the C-terminal of PIEZO2, which results in increased channel activity. Nevertheless, all of the recessive inherited PIEZO2-related disorders are caused by biallelic truncating mutations which may induce nonsense-mediated decay of the mRNA [21] (Table 3) (Fig. 4d). As GoF and LoF variants in PIEZO2 have different effects on muscle development and disease pathophysiology, different inheritance modes and mutation types lead to distinct DA phenotypes.

\section{Conclusions}

In conclusion, we firstly reported two novel mutations within TPM2 and PIEZO2 causing DA2B and mild DA in two Chinese families, respectively. Our study also expands the spectrum of causal mutations in the TPM2 and PIEZO2 genes.

\section{Additional files}

Additional file 1: Table S1. Primer used in this study (XLS 10 kb)

Additional file 2: Figure S1. Ultrasound examination of a fetus (IV: 1) at 22 weeks of gestational age. (A) Arrow indicates extended wrist and clenched hand. Polyhydramnios is apparent. (B) Arrow specifies bilateral clubfoot with a deformity of the toes (PPT $329 \mathrm{~kb}$ )

Additional file 3: Table S2. Two-point linkage analysis using 3 genetic markers in the DA2B family (XLSX 9 kb)

\section{Abbreviations}

DA: Distal arthrogryposis; HGMD: Human Gene Mutation Database;

LOD: Logarithm of odds; PCR-RFLP: Polymerase chain reaction-restriction fragment length polymorphism; PROVEAN: Protein variation effect analyzer; SIFT: Scale-invariant feature transform; WES: Whole-exome sequencing

\section{Acknowledgements}

We are very grateful to the family members for their participation in this study, as well as the support of CAMS Innovation Fund for Medical Sciences.

\section{Funding}

This study was supported by the CAMS Innovation Fund for Medical Sciences (CIFMS) (2016-12M-3-003) and the National Key Research and Development Program of China (2016YFE0128400 and 2016YFC0905100).

\section{Availability of data and materials}

The datasets used and/or analysed during the current study are available from the corresponding author on reasonable request.

\section{Authors' contributions}

SL and YY conducted experiments, data analysis and drafting the manuscript. JG helped with clinical assessment of patients. BM helped to write the final manuscript. $Y C$ renewed the patient information. $X L Z$ and $X Z$ designed and supervised the study. All authors read and approved the final manuscript.

\section{Ethics approval and consent to participate}

This study was approved by the Institutional Review Board (IRB) of the Institute of Basic Medical Sciences, Chinese Academy of Medical Sciences, Beijing, China (015-2015). Written informed consent was obtained from the patients or their relatives before they participated in this study.

\section{Consent for publication}

Written consent was obtained from the patients or their relatives for the publication of this study, including the medical data in Table 1 and the images in Fig. 2.

Competing interests

The authors declare that they have no competing interests.

\section{Publisher's Note}

Springer Nature remains neutral with regard to jurisdictional claims in published maps and institutional affiliations. 


\section{Author details}

'Department of Medical Genetics, Institute of Basic Medical Sciences, Chinese Academy of Medical Sciences \& School of Basic Medicine, Peking Union Medical College, 5 Dong Dan San Tiao, Dong Cheng District, Beijing 100005, China. ${ }^{2}$ Department of Obstetrics and Gynecology, Peking Union Medical College Hospital, Beijing 100730, China.

Received: 19 April 2018 Accepted: 23 September 2018

Published online: 03 October 2018

\section{References}

1. Hall JG, Reed SD, Greene G. The distal arthrogryposes - delineation of new entities - review and Nosologic discussion. Am J Med Genet. 1982;11(2): 185-239.

2. Bamshad M, Van Heest AE, Pleasure D. Arthrogryposis: a review and update. J Bone Joint Surg Am. 2009;91(Suppl 4):40-6.

3. Krakowiak PA, Bohnsack JF, Carey JC, Bamshad M. Clinical analysis of a variant of freeman-Sheldon syndrome (DA2B). Am J Med Genet. 1998;76(1):93-8.

4. Bamshad M, Watkins WS, Zenger RK, Bohnsack JF, Carey JC, Otterud B, Krakowiak PA, Robertson M, Jorde LB. A gene for distal arthrogryposis type I maps to the pericentromeric region of chromosome 9. Am J Hum Genet. 1994:55(6):1153-8.

5. Kimber E. AMC: amyoplasia and distal arthrogryposis. J Child Orthop. 2015; 9(6):427-32.

6. O'Shaughnessy KM. Gordon syndrome: a continuing story. Pediatr Nephrol. 2015:30(11):1903-8.

7. Gurnett CA, Alaee F, Desruisseau D, Boehm S, Dobbs MB. Skeletal muscle contractile gene (TNNT3, MYH3, TPM2) mutations not found in vertical talus or clubfoot. Clin Orthop Relat Res. 2009;467(5):1195-200.

8. McMillin MJ, Beck AE, Chong JX, Shively KM, Buckingham KJ, Gildersleeve HI, Aracena MI, Aylsworth AS, Bitoun P, Carey JC, et al. Mutations in PIEZO2 cause Gordon syndrome, Marden-Walker syndrome, and distal arthrogryposis type 5. Am J Hum Genet. 2014;94(5):734-44.

9. Manzin A, Salvoni G, Bagnarelli P, Menzo S, Carloni G, Clementi M. A singlestep DNA extraction procedure for the detection of serum hepatitis B virus sequences by the polymerase chain reaction. J Virol Methods. 1991;32(2-3): 245-53.

10. Jiang $M$, Zhao X, Han W, Bian C, Li X, Wang G, Ao Y, Li Y, Yi D, Zhe Y, et al. A novel deletion in TNNI2 causes distal arthrogryposis in a large Chinese family with marked variability of expression. Hum Genet. 2006;120(2):238-42.

11. Wei X, Ju X, Yi X, Zhu Q, Qu N, Liu T, Chen Y, Jiang H, Yang G, Zhen R, et al. Identification of sequence variants in genetic disease-causing genes using targeted next-generation sequencing. PLoS One. 2011;6(12):e29500.

12. Tajsharghi H, Ohlsson M, Palm L, Oldfors A. Myopathies associated with beta-tropomyosin mutations. Neuromuscul Disord. 2012;22(11):923-33.

13. Sung SS, Brassington AM, Grannatt K, Rutherford A, Whitby FG, Krakowiak PA, Jorde LB, Carey JC, Bamshad M. Mutations in genes encoding fasttwitch contractile proteins cause distal arthrogryposis syndromes. Am J Hum Genet. 2003;72(3):681-90.

14. Tajsharghi $H$, Kimber $E$, Holmgren D, Tulinius M, Oldfors A. Distal arthrogryposis and muscle weakness associated with a beta-tropomyosin mutation. Neurology. 2007;68(10):772-5.

15. Beck AE, McMillin MJ, Gildersleeve HI, Kezele PR, Shively KM, Carey JC, Regnier M, Bamshad MJ. Spectrum of mutations that cause distal arthrogryposis types 1 and 2B. Am J Med Genet A. 2013;161A(3):550-5.

16. Ohlsson M, Quijano-Roy S, Darin N, Brochier G, Lacene E, Avila-Smirnow D, Fardeau M, Oldfors A, Tajsharghi H. New morphologic and genetic findings in cap disease associated with beta-tropomyosin (TPM2) mutations. Neurology. 2008;71(23):1896-901.

17. Jarraya M, Quijano-Roy S, Monnier N, Behin A, Avila-Smirnov D, Romero NB, Allamand V, Richard P, Barois A, May A, et al. Whole-body muscle MRI in a series of patients with congenital myopathy related to TPM2 gene mutations. Neuromuscul Disord. 2012;22(Suppl 2):S137-47.

18. Karpicheva OE, Redwood CS, Borovikov YS. The E117K mutation in betatropomyosin disturbs concerted conformational changes of actomyosin in muscle fibers. Arch Biochem Biophys. 2014;549:12-6.

19. Mroczek M, Kabzinska D, Chrzanowska KH, Pronicki M, Kochanski A. A novel TPM2 gene splice-site mutation causes severe congenital myopathy with arthrogryposis and dysmorphic features. J Appl Genet. 2017;58(2):199-203.

20. Marttila M, Lehtokari V-L, Marston S, Nyman TA, Barnerias C, Beggs AH, Bertini E, Ceyhan-Birsoy Ö, Cintas P, Gerard M, et al. Mutation update and genotype-phenotype correlations of novel and previously described mutations inTPM2andTPM3Causing congenital myopathies. Hum Mutat. 2014;35(7):779-90

21. Delle Vedove A, Storbeck M, Heller R, Holker I, Hebbar M, Shukla A, Magnusson O, Cirak S, Girisha KM, O'Driscoll M, et al. Biallelic loss of proprioception-related PIEZO2 causes muscular atrophy with perinatal respiratory distress, arthrogryposis, and scoliosis. Am J Hum Genet. 2016; 99(5):1206-16.

22. Okubo M, Fujita A, Saito Y, Komaki H, Ishiyama A, Takeshita E, Kojima E, Koichihara R, Saito T, Nakagawa E, et al. A family of distal arthrogryposis type 5 due to a novel PIEZO2 mutation. Am J Med Genet A. 2015;167A(5): 1100-6.

23. Coste B, Houge G, Murray MF, Stitziel N, Bandell M, Giovanni MA, Philippakis A, Hoischen A, Riemer G, Steen U, et al. Gain-of-function mutations in the mechanically activated ion channel PIEZO2 cause a subtype of distal arthrogryposis. Proc Natl Acad Sci U S A. 2013;110(12):4667-72.

24. Harris E, Topf A, Barresi R, Hudson J, Powell H, Tellez J, Hicks D, Porter A, Bertoli $M$, Evangelista $T$, et al. Exome sequences versus sequential gene testing in the UK highly specialised Service for Limb Girdle Muscular Dystrophy. Orphanet J Rare Dis. 2017;12(1):151.

25. Chesler AT, Szczot M, Bharucha-Goebel D, Ceko M, Donkervoort S, Laubacher C, Hayes LH, Alter K, Zampieri C, Stanley C, et al. The role of PIEZO2 in human Mechanosensation. N Engl J Med. 2016;375(14):1355-64.

26. Haliloglu G, Becker K, Temucin C, Talim B, Kucuksahin N, Pergande M, Motameny S, Nurnberg P, Aydingoz U, Topaloglu H, et al. Recessive PIEZO2 stop mutation causes distal arthrogryposis with distal muscle weakness, scoliosis and proprioception defects. J Hum Genet. 2017;62(4):497-501.

27. Mahmud AA, Nahid NA, Nassif C, Sayeed MS, Ahmed MU, Parveen M, Khalil MI, Islam MM, Nahar Z, Rypens F, et al. Loss of the proprioception and touch sensation channel PIEZO2 in siblings with a progressive form of contractures. Clin Genet. 2017;91(3):470-5.

28. Alfares A, Alfadhel M, Wani T, Alsahli S, Alluhaydan I, Al Mutairi F, Alothaim A, Albalwi M, Al Subaie L, Alturki S, et al: A multicenter clinical exome study in unselected cohorts from a consanguineous population of Saudi Arabia demonstrated a high diagnostic yield. Mol Genet Metab. 2017;121(2):91-95.

29. Monies D, Abouelhoda M, AlSayed M, Alhassnan Z, Alotaibi M, Kayyali H, AlOwain M, Shah A, Rahbeeni Z, Al-Muhaizea MA, et al: The landscape of genetic diseases in Saudi Arabia based on the first 1000 diagnostic panels and exomes. Hum Genet. 2017;136(8):921-39.

30. Alisch F, Weichert A, Kalache K, Paradiso V, Longardt AC, Dame C, Hoffmann K, Horn D: Familial Gordon syndrome associated with a PIEZO2 mutation. Am J Med Genet A. 2017;173(1):254-59.
Ready to submit your research? Choose BMC and benefit from:
- fast, convenient online submission
- thorough peer review by experienced researchers in your field
- rapid publication on acceptance
- support for research data, including large and complex data types
- gold Open Access which fosters wider collaboration and increased citations
- maximum visibility for your research: over $100 \mathrm{M}$ website views per year
At BMC, research is always in progress.
Learn more biomedcentral.com/submissions 